\title{
HERRAMIENTA DIDÁCTICA PARA EL DESARROLLO Y PERFECCIONAMIENTO DE HABILIDADES SUPERIORES DE PENSAMIENTO
}

\author{
Margarita Eugenia Laisequilla Rodríguez \\ Universidad Internacional Iberoamericana UNINI (México)
}

\begin{abstract}
Resumen. La Educación debe llevar al alumnado a elevar sus niveles de razonamiento para ayudarlos en el desarrollo de un pensamiento de carácter superior. No obstante, esto no ocurre cotidianamente en las aulas. En México, conforme se avanza en los niveles educativos, se incrementa en los niños la carencia del nivel de pensamiento correspondiente a su edad. Han existido diversos programas para ayudar a los niños a desarrollar su pensamiento que han evidenciado la necesidad que tienen de recibir un andamiaje para alcanzar su máximo potencial en dichas habilidades. Los maestros deben ayudar a sus alumnos brindándoles experiencias enriquecidas, guiándolos hacia la reflexión. El pensamiento reflexivo hace que los alumnos razonen de manera superior logrando aplicar sus conocimientos. El objetivo fue conocer la forma en que ocurren avances en el desarrollo del pensamiento en los niños de tercer año de Secundaria (14 y 15 años de edad) al brindarles el espacio y la guía necesarios para la reflexión. Elegimos una metodología cualitativa para así conocer directamente de los niños la forma en la que fueron desarrollando sus habilidades de pensamiento, así como la influencia de la mediación docente. Durante la investigación, los alumnos resolvieron reactivos de las pruebas PISA que evalúan su capacidad para transferir los conocimientos a contextos reales, considerado esto como una habilidad superior de pensamiento, realizando, además, ejercicios de reflexión y metacognición. Los resultados mostraron grandes avances en la capacidad de pensamiento de los alumnos, mejorando además su desempeño escolar y su nivel de acuerdo a PISA.
\end{abstract}

Palabras clave: andamiaje, habilidades superiores de pensamiento, mediación docente, metacognición, pensamiento reflexivo.

\section{DIDACTIC TOOL FOR THE DEVELOPMENT AND PERFECTION OF HIGHER ORDER THINKING SKILLS}

\begin{abstract}
Education must lead the student body to increase its reasoning levels to help them in the development of higher order thinking. Nonetheless, this does not occur daily in the classrooms. In Mexico, as education levels advance, the scarcity of the thinking level corresponding to their age increases in children. There have existed diverse programs to help children develop their thinking that have evidenced their need to receive a scaffolding to reach their maximum potential in such skills. Teachers have the responsibility to help their students providing them enriched experiences, guiding them to reflection. Reflective thinking makes students reason in a superior way, so that they achieve to apply their knowledge. The objective was to know the way in which progress is being reached in the development of thinking skill in 9th grade children (14 to 15 years old) providing them the space and guidance necessary for reflection. We chose a qualitative methodology to get to know directly from the children the way in which they were developing their thinking skills, as well as the influence of teaching mediation. During the investigation students solved questions of the PISA tests that evaluate their capacity to transfer their knowledge to real contexts, considering this as a high thinking skill, also carrying out reflection exercises and metacognition. The results showed great progress in the thinking capacity of the students, improving also their school performance and their level according to PISA.
\end{abstract}


Key words: higher order thinking skills, metacognition, reflective thinking, scaffolding, teaching mediation.

\section{FERRAMENTA DIDÁTICA PARA O DESENVOLVIMENTO E APERFEIÇOAMENTO DE HABILIDADES DE PENSAMENTO SUPERIORES}

Resumo. A Educação deve levar os alunos a elevar seus níveis de raciocínio para auxiliá-los no desenvolvimento de um pensamento de caráter superior. No entanto, isso não ocorre diariamente na sala de aula. No México, à medida que avançamos nos níveis educativos, aumenta nas crianças a falta de um nível de pensamento que corresponda à idade. Existiram vários programas para ajudar as crianças no desenvolvimento do pensamento que evidenciaram a necessidade de receber uma estrutura para que consigam atingir seu pleno potencial nessas habilidades. Os professores têm a responsabilidade de ajudar seus alunos, lhes proporcionando experiências ricas e orientando-os para a reflexão. O pensamento reflexivo faz com que os alunos raciocinem de forma superior e consigam aplicar seus conhecimentos. $\mathrm{O}$ objetivo era saber de que forma se conseguem avanços no desenvolvimento de habilidades de pensamento em crianças do terceiro ano do Ensino Secundário (14 e 15 anos de idade), oferecendo espaços e orientações para a reflexão. Escolhemos uma metodologia qualitativa para conhecermos diretamente dos alunos a forma como foram desenvolvendo suas habilidades de pensamento, bem como a influência da mediação docente. Durante a investigação, os alunos resolveram questões dos testes PISA, que avaliam sua capacidade de transferir conhecimentos para contextos reais, sendo isso considerado uma habilidade superior de pensamento, além de exercícios de reflexão e metacognição. Os resultados mostraram grandes progressos na capacidade de pensamento dos alunos, além de melhoras no desempenho escolar e em seu nível de acordo com o PISA.

Palavras-chave: estrutura, habilidades superiores de pensamento, mediação docente, metacognição, pensamento reflexivo.

\section{Introducción}

Desde la antigüedad, filósofos griegos afirmaron que la Educación debía enseñar a los jóvenes a pensar, reflexionar y razonar (Moore, 2006). Debido a la preocupación surgida por los bajos resultados obtenidos en México en pruebas tanto internacionales como nacionales aplicadas a niños de quince años, lo que denota una deficiencia en sus habilidades de pensamiento, surge la idea de realizar la presente investigación que tiene como objetivo general proponer una herramienta didáctica que pueda integrarse al currículum actual existente en tercer año de Secundaria en México, con el propósito de ayudar a perfeccionar las habilidades superiores de pensamiento de los alumnos.

El Instituto Nacional para la Evaluación de la Educación (INEE) en México reporta que conforme se avanza en los niveles escolares, es más grande el problema existente de adquisición de pensamiento matemático en los niños, así como en el área de Español. Menciona que a nivel nacional, el nueve por ciento de niños de tercer curso de Preescolar no desarrolla las competencias correspondientes a su edad en ninguna de las dos áreas; en la misma situación aparece el diecisiete por ciento de los alumnos de Sexto de Primaria, mientras que el problema se agudiza en alumnos de tercer curso de Secundaria ya que dicho porcentaje se eleva a un cincuenta y uno por ciento (Larrazolo, Backhoff, y Tirado, 2013). 
Las investigaciones realizadas por educadores e investigadores en el área de Educación, como las de Feurestein (Nickerson, Perkins, \& Smith, 1985), Lipman (1998), Costa (2001a) y González-Moreno (2012), han mostrado un interés especial y progresivo en el tema del desarrollo de las habilidades de pensamiento; algunas de sus propuestas han consistido en programas completos para enseñar a pensar. Sin embargo, siguiendo a Saiz (2002), solo se puede enseñar a los alumnos a pensar mediante la práctica y esto no es algo que ocurra normalmente en las aulas, por lo cual los jóvenes entran a las universidades con niveles de pensamiento no aptos para su futuro desarrollo profesional y personal.

\section{Habilidades superiores de pensamiento}

Lipman (1998) afirma que el origen del problema ocurre en las escuelas y se debe a que los alumnos no reflexionan sobre sus conocimientos, o lo hacen muy poco. Esto pudiera llevarlos en un futuro a ser ciudadanos poco reflexivos y productivos.

Es importante mencionar que las habilidades superiores de pensamiento son el producto de un proceso de desarrollo que no ocurre como resultado de la maduración (Case, 1992, citado por Beyer, 2001). Además, tampoco es algo que ocurra como consecuencia del aprendizaje de las asignaturas, sino que requiere tiempo y experiencia. Las habilidades superiores de pensamiento son procesos mentales que constan de complicados procedimientos que utilizan habilidades como análisis, síntesis, comparación, inferencia, interpretación, evaluación y razonamientos inductivo y deductivo para la resolución de nuevos problemas o situaciones (Budsankom, Sawangboon, Damrongpanit \& Chuensirimongkol, 2015).

El pensamiento es un elemento que se encuentra presente en todo aprendizaje significativo, por lo cual debiera ser algo que se enseñe en las aulas desde la Educación Preescolar; Muria y Damián (2008) afirman que el pensamiento es algo susceptible de perfeccionarse a lo largo de la vida.

Han existido diversos programas para enseñar a pensar que han plasmado de manera contundente la necesidad que tienen los niños y jóvenes de recibir un andamiaje para lograr su máximo potencial en habilidades de pensamiento. Presentamos a continuación algunos que consideramos relevantes.

El Programa de enriquecimiento instrumental de Feurestein es uno de los pocos que han sido utilizados y evaluados ampliamente (Nickerson et al., 1985). Este autor afirma que los niños presentan grandes avances en sus niveles de pensamiento cuando sus padres o profesores median entre el niño y el mundo por medio del refuerzo de estímulos para lograr avances en su desarrollo cognitivo.

El Programa de Filosofía para Niños de Lipman (Nickerson et al., 1985) se centra en el fomento y el desarrollo de las habilidades de razonamiento filosófico. Entre las habilidades que se logra desarrollar en los alumnos se encuentra poder realizar inferencias, asociaciones y analogías, formular hipótesis, elaborar distinciones significativas, considerar alternativas, así como buscar razones, explicaciones y supuestos. Como resultado de estudios realizados a este programa, se han encontrado mejorías en el desempeño intelectual de los alumnos, especialmente en lectura, matemáticas y en razonamiento creativo y formal.

El Proyecto Inteligencia surgió con el objetivo de mejorar el pensamiento y la inteligencia de individuos de todas las edades, niveles educativos y áreas de trabajo. Los beneficios de los alumnos que han participado en este programa fueron probados con 
diferencias estadísticamente significativas y de magnitud sustancial con relación a alumnos que habían recibido una educación tradicional (Sánchez, 2002).

\section{El pensamiento como vehículo para la comprensión y el aprendizaje}

Además de las habilidades superiores mencionadas, existen dos, también consideradas como superiores, que los alumnos necesitarán desarrollar durante su etapa escolar para lograr tomar el control de su aprendizaje y para poder aplicar sus conocimientos en otras áreas de estudio o en la vida real. Estas dos habilidades referidas son la metacognición y la transferencia.

La metacognición se refiere al conocimiento acerca de los propios procesos de pensamiento (Flavell 1976, citado por Presseisen, 2001). Los aprendices deben monitorear el uso de sus procesos de pensamiento y regularlos de acuerdo a sus objetivos cognitivos. Algunas actividades metacognitivas incluyen la búsqueda de errores, la revisión de la comprensión, releer algunos pasajes o volver a realizar algunos cálculos (Presseisen, 2001). Las investigaciones realizadas en relación a la metacognición reconocen que la instrucción y la guía para el control y regulación de los procesos de pensamiento ha mostrado un fuerte impacto en los logros alcanzados (Paris, Wasik \& Turner, 1991; Schoenfeld, 1992, citados por Marzano, 2001).

La transferencia también es considerada como una habilidad metacognitiva (Nickerson et al., 1985), y por lo tanto, de alto nivel. Perkins y Salomon (2001) han observado que es difícil que las personas transfieran habilidades de pensamiento entre un contexto y otro sin necesidad de ayuda. Estos autores mencionan que para lograr esta transferencia los profesores deben hacer que los alumnos apliquen habilidades de pensamiento en un contexto diferente al original en el que estas fueron aprendidas. Para ello, también es necesario que los alumnos aprendan a descubrir las similitudes entre diferentes contextos para que más adelante sean capaces de identificar otras áreas en las cuales dicha habilidad sea aplicable.

Para comprobar el beneficio de una instrucción en la que se enfatice el pensamiento de alto nivel, Wenglinsky (2004, citado por Brookhart, 2010), realizó una revisión de estudios en los que se haya intentado relacionar dicha instrucción con un desempeño superior, y como resultado, el investigador reportó que tanto en los exámenes National Assessment of Educational Progress y en las pruebas Trends in International Mathematics and Science Study, los resultados fueron contundentes. En Matemáticas y en Ciencias se logró relacionar la enseñanza basada en el razonamiento con mejores calificaciones en alumnos de todos los niveles evaluados. En Lectura se logró relacionar la enseñanza orientada hacia la construcción de significados con mejores desempeños de los alumnos.

Pensamos que el diseño curricular que existe actualmente en diversas partes del mundo, incluyendo México (SEP, 2011), invita a los docentes a ayudar a sus alumnos a desarrollar habilidades de pensamiento. Muchos de los objetivos curriculares inician con verbos que implican acciones que exigen de los alumnos determinadas habilidades de pensamiento como analizar, comparar, etc. Sin embargo, los bajos resultados de los alumnos sugieren que los docentes se han estado enfocando en abarcar los contenidos del plan de estudios y no en que los alumnos aprendan a analizar, comparar, o cualquier otra habilidad de pensamiento, como si esto fuera a ser una consecuencia directa de la enseñanza de los contenidos. Resnick (2001) enfatiza que se debe trabajar mucho en desarrollar diferentes maneras de lograr el desarrollo de habilidades de pensamiento de los alumnos dentro de las disciplinas académicas. 


\section{El pensamiento reflexivo para el desarrollo de las habilidades superiores de pensamiento}

De acuerdo a González-Moreno (2012, p. 597), "la formación del pensamiento reflexivo es una necesidad en la sociedad actual. Pensar reflexivamente permite acceder con facilidad a los diferentes campos del saber". Cuando un alumno piensa reflexivamente, aprende, hace asunciones, juzga y logra aplicar el conocimiento. Esta actividad de pensamiento se convierte en una comprensión profunda, el pensamiento cambia. Hmelo y Ferrari (1997) han concluido que la reflexión ayuda a los alumnos a construir habilidades de pensamiento de alto nivel.

La reflexión es considerada como una de las estrategias de aprendizaje más poderosas. La mente de una persona que reflexiona se encuentra en una disposición especial que puede llevarlo a desarrollar grandes ideas y soluciones, así como a discernir el conocimiento que desea adquirir. "El pensamiento reflexivo, que es el compromiso mental durante el proceso cognitivo para comprender los factores conflictivos en una situación, es considerado como un componente crítico dentro del proceso de aprendizaje" (Song, Koszalka \& Grabowski, 2005).

En 2015 fue realizada una investigación cuyo objetivo fue conocer el mejoramiento en la habilidad de razonamiento matemático en los alumnos por medio del aprendizaje reflexivo (Rohana, 2015). La investigación se realizó con ciento cincuenta y cinco alumnos de nivel universitario. Como resultado de dicho estudio, los autores concluyeron que el aprendizaje reflexivo tuvo una influencia determinante en el desarrollo y mejoramiento de las habilidades de razonamiento matemático en los estudiantes.

\section{Método}

Para la investigación elegimos un enfoque cualitativo ya que siguiendo a Quinn (1990), es el idóneo cuando se intenta conocer lo que las personas hacen, saben y piensan, utilizando la observación, las entrevistas y el análisis de documentos. En este caso lo importante es el proceso del desarrollo cognitivo de los niños. También utilizamos instrumentos cuantitativos para la recolección de información, a manera de apoyo, lo cual es válido de acuerdo a Hernández, Fernández-Collado, y Baptista (2008) quienes mencionan que "en el levantamiento de datos cualitativos podría utilizarse una herramienta cuantitativa".

La muestra fue el total de alumnos inscritos en tercer año de Secundaria en la Institución donde obtuvimos los permisos necesarios. Este tipo de muestreo se denomina muestreo por conveniencia, en donde se eligen aquellos casos a los que se puede tener acceso (Hernández et al., 2008).

El número de alumnos inscritos en dicho nivel fue de ciento sesenta y dos. Siguiendo a Hernández et al. (2008), para la investigación cualitativa el tamaño de la muestra no es importante desde una perspectiva probabilística, ya que el interés del investigador no es generalizar los resultados de su estudio, sino profundizar. siguientes:

Los instrumentos seleccionados para la recolección de datos fueron los 
Observación participante. Realizamos observaciones de las sesiones en el aula en donde los alumnos llevaron a cabo un debate reflexivo y metacognitivo.

Análisis de documentos. Los documentos analizados son formatos que diseñamos para que los alumnos plasmaran por escrito sus procesos de pensamiento, reflexivos y metacognitivos, inmediatamente después de haber respondido el reactivo de PISA correspondiente. Esta información fue utilizada además, para ir elaborando la herramienta de perfeccionamiento de las habilidades de pensamiento en los alumnos.

Entrevistas de profundidad. Al final de la investigación realizamos una entrevista a profundidad a las tres docentes participantes para que hablaran abiertamente sobre determinados tópicos relacionados con el trabajo realizado con los alumnos y además para que respondieran algunas preguntas de forma totalmente libre. Estos tópicos tienen el propósito de conocer su percepción sobre su propia mediación para el aprendizaje de los alumnos y los resultados de esta, así como del desarrollo de las habilidades de pensamiento que hayan observado en sus alumnos durante el curso de la investigación.

El procedimiento durante el trabajo de campo inició con la aplicación de la prueba inicial de diagnóstico con reactivos PISA abarcando las competencias científica, matemática y de lectura. Con esta información se pudieron conocer las capacidades de pensamiento de los alumnos en dicho momento siendo posible ubicar a cada niño en determinado nivel de desempeño.

Posteriormente, los alumnos respondieron durante veintiún días un reactivo diferente por sesión. Estas sesiones tuvieron una duración de treinta minutos, iniciando con la resolución del reactivo correspondiente. Durante estas sesiones, los alumnos llevaron a cabo un ejercicio de reflexión metacognitiva sobre cada una de las preguntas del reactivo, respondiendo el Ejercicio de procesos de pensamiento, para externar el pensamiento y razonamiento que usaron durante la resolución de las preguntas (Figura $1)$.

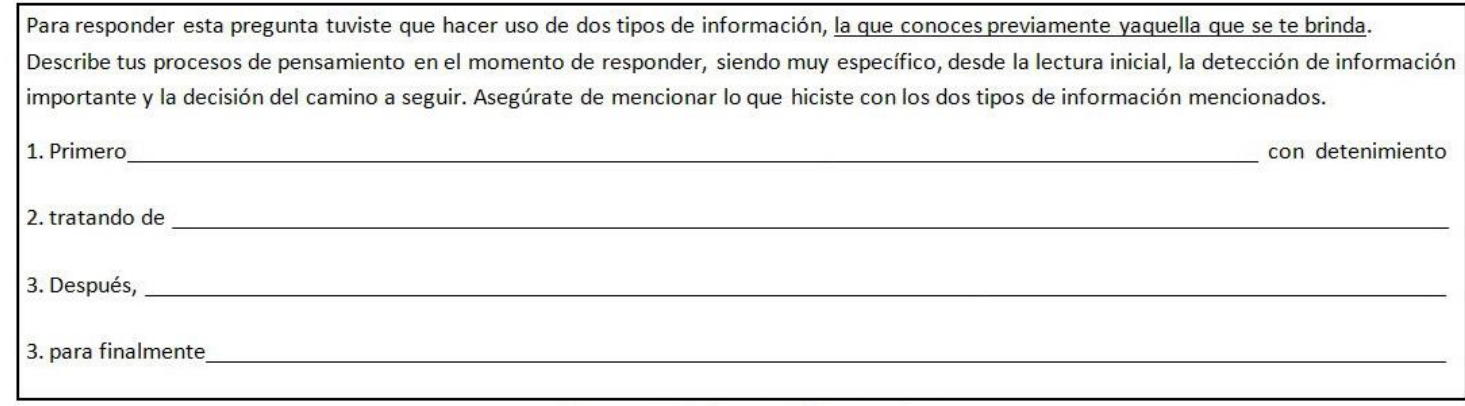

Figura 1. Ejercicio de procesos de pensamiento.

Después, a los alumnos les fue comunicada la respuesta correcta a cada pregunta así como una manera de razonamiento que los hubiera llevado a dicha resolución, para que reflexionaran sobre la forma, igual o distinta, en la que ellos hayan razonado durante el proceso. Este Ejercicio metacognitivo para el aprendizaje por medio de la concientización de errores aparece en la Figura 2; el último punto de este ejercicio es que los alumnos comunicaran sus aprendizajes alcanzados. 


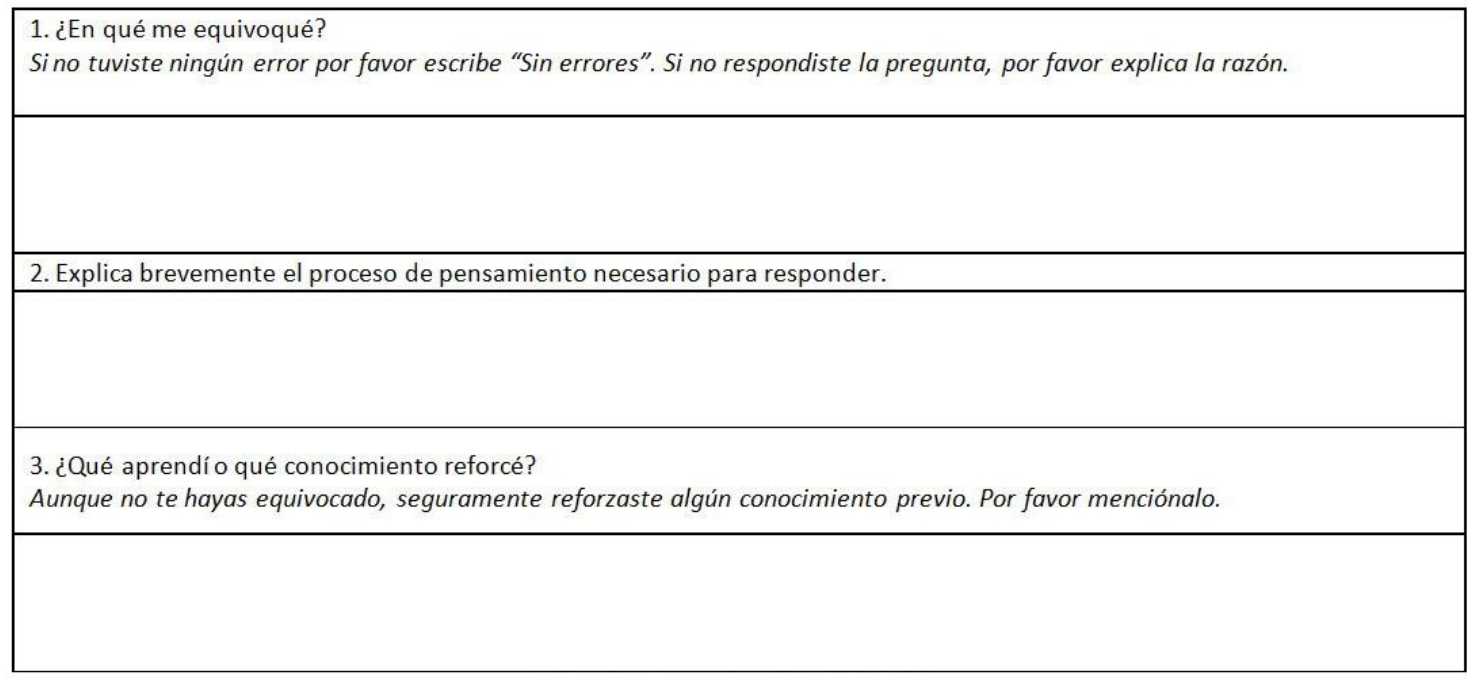

Figura 2. Ejercicio metacognitivo para el aprendizaje por medio de la concientización de los errores

Finalmente, los alumnos respondieron los mismos reactivos de la prueba inicial, con el propósito de encontrar alguna diferencia entre ambos.

Para el análisis de los datos, estos fueron clasificados de acuerdo a tres categorías de análisis que fueron definidas con base en los constructos considerados los más relevantes de la investigación: Desarrollo del pensamiento superior en los niños, Mediación docente favorecedora de un pensamiento superior y Nivel de desempeño final de los niños.

\section{Resultados}

Los resultados se presentan por categoría de análisis:

\section{Primera categoría: Desarrollo del pensamiento superior en los niños}

Después de las veintiuna sesiones dedicadas al desarrollo de las capacidades de pensamiento de los alumnos, pudimos observar grandes avances por medio de las reflexiones realizadas. Como afirman Hmelo y Ferrari (1997), al reflexionar, los alumnos construyen habilidades de pensamiento de alto nivel.

Los niños fueron aprendiendo a pensar de manera superior y a identificar sus pensamientos. Brooks y Brooks (2001) enfatizan que en el aula se utilice un lenguaje determinado para referirse a procesos de pensamiento, con el objetivo de que se convierta en un hábito y que los alumnos se acostumbren a escucharlo y a utilizarlo. Para ello, desde el inicio de las sesiones, se les entregó una lista con verbos que representan actividades mentales superiores incluyendo sus significados.

Dentro de los avances observados en los alumnos para explicar sus procesos de pensamiento encontramos respuestas metacognitivas como en el reactivo Lluvia ácida, que trata de unas estatuas que estaban siendo corroídas por la lluvia ácida, donde un alumno mencionó: "inferí que el mármol se iba a corroer", o en el reactivo Crecimiento, que presenta una gráfica con las diferencias entre el crecimiento de hombres y mujeres, donde otro alumno comunicó: "deduje que al ser la pendiente menor, eran menos centímetros por año". También observamos dichos procesos 
superiores aunque los niños no mencionaran el término correspondiente; por ejemplo, la habilidad superior de secuenciación, que implica el reconocimiento de patrones (Argüelles y Nagles, 2000) fue utilizada por muchos niños para responder uno de los reactivos de la competencia matemática Manzanas, en el que tenían que realizar una secuencia para predecir cuál número de árboles, entre coníferas y manzanos sembrados en una granja, crecería más rápido. Otro ejemplo es el del verbo conceptualizar, proceso que implica la construcción de una representación mental del objeto o situación (Argüelles y Nagles, 2000); aunque este verbo no fue mencionado por los alumnos, esta capacidad sí fue demostrada tanto en los ejercicios escritos como durante los foros de discusión.

Durante las sesiones constatamos una mejoría progresiva en la calidad mostrada en las respuestas de los alumnos. En ocasiones hacían mención de algún dato o proceso que ayudaba a la comprensión de la situación y en ocasiones expresaban una idea importante para llegar a la respuesta, justo como Dewey (2007) define el pensamiento reflexivo. Al mencionar calidad nos referimos a elementos que denoten un pensamiento superior.

No fue nuestro objetivo que los alumnos mejoraran su lenguaje, aunque las maestras comentaron este beneficio, mencionando que el vocabulario de los alumnos al principio era muy reducido y posteriormente observábamos respuestas como“...deduje mi respuesta a partir del planteamiento del problema". En este sentido, tanto Brooks y Brooks (2001) como Costa (2001b) afirman que los niños en el aula deben pensar en términos de verbos que representen una actividad mental superior, como "predecir", en lugar de "va a ocurrir". Esto tiene el propósito de ayudarlos en el desarrollo de su metacognición, lo cual los lleva al desarrollo de otras habilidades superiores de pensamiento (Flavell, 1979, citado por Correa, Castro, y Lira, 2002).

$\mathrm{Al}$ inicio de las sesiones reflexivas, los pensamientos de los alumnos no siempre los ayudaban a responder acertadamente la pregunta. Sin embargo, los alumnos mejoraron sustancialmente observándose después en sus reflexiones procesos superiores de pensamiento. Mencionaremos a continuación algunas respuestas superiores brindadas por los alumnos durante los ejercicios escritos o en los foros de discusión. En dichas respuestas mencionan, por ejemplo, la manera en la que pensaron para llegar a la respuesta en alguna pregunta determinada, su proceso de pensamiento cuando compararon su respuesta con la respuesta correcta o bien, el aprendizaje final alcanzado.

Lo más notorio al principio fue que los niños fueron aprendiendo a identificar y a nombrar sus procesos de pensamiento. Durante la resolución del reactivo Donación de sangre, en el cual se presenta al alumno un artículo acerca de algunos aspectos a considerar antes de donar, fue muy satisfactorio identificar respuestas de los alumnos con verbos que expresaban capacidades superiores de pensamiento correctamente aplicadas como: "Primero analicé el texto poco a poco y reflexioné por qué son estériles. Después recordé cuando me sacaron sangre y analicé para concluir que lo hacían para certificar la donación de sangre", en donde el alumno además de enfocarse en la palabra estériles, comunica la reflexión que realizó para decidir la razón por la cual en la pregunta era remarcado este término. Además, también mostró una transferencia de conocimientos ya que recordó cuando a él se sacaron sangre, llegando en su mente a concluir sobre la importancia de certificar la donación de sangre.

En relación a la reflexión realizada por los alumnos para identificar sus procesos de pensamiento, una de las respuestas encontradas que más elementos metacognitivos expresa es la siguiente, realizada durante en el reactivo de Triángulos en el cual los 
alumnos tenían que identificar uno entre varios triángulos que cumpliera con una lista de características: "Primero observé la imagen y decidí utilizar un método de descarte, tratando primeramente de eliminar aquellas figuras que no parecían tener 90 grados (E y D). Después busqué que su ángulo recto estuviera en $\mathrm{R}$ y descarté el triángulo $\mathrm{C}$, para finalmente darme cuenta que el triángulo D también era de 90 grados y cumplía con la característica de que el punto S se hallaba dentro del triángulo y todas las demás". En su respuesta el alumno menciona haber utilizado la habilidad de descartar, que era la indicada, describiendo los pasos realizados hasta determinar el triángulo correcto. Bereiter (1980, citado por Nickerson et al., 1985) menciona la importancia de enseñar a los estudiantes a pensar sobre el papel, y esto fue lo que provocamos en los alumnos logrando los avances mencionados.

Encontramos asimismo elementos metacognitivos durante los foros de discusión, como el realizado durante el reactivo Lluvia ácida, ya mencionado, donde un alumno comentó: "Relacioné que el agua corroe algunos materiales. Pensé que el vinagre es ácido y también corroe"; esta respuesta aunque es breve refleja un pensamiento superior. También durante el reactivo El lago Chad, en el que se muestran las fluctuaciones del nivel del lago ubicado en el Sahara, así como pinturas rupestres encontradas alrededor del lago y cambios de patrones en la vida salvaje, observamos respuestas reflexivas como: "Primero revisé las opciones y leí la pregunta y me decía que por qué habían plasmado a los animales. Cuando leí las preguntas pensé: No puede ser que los pintores caminen mucho porque no tiene sentido, no llevan al animal con ellos, y luego pensé que no podían ser las otras porque no tenían mucho sentido. Cuando leí esa que decía que era porque estaban ahí los animales, lo relacioné con la lectura y se me hizo más lógico que si estaban ahí hayan tomado inspiración y hayan querido plasmar lo que estaban viendo en ese momento". En esta respuesta el alumno comunica muy asertivamente lo que iba ocurriendo en su mente durante la reflexión realizada.

El gran avance logrado en el pensamiento reflexivo de los alumnos pudo observarse en los foros con aportaciones en las que mencionaban cómo llegaron a solucionar un problema o situación. En el último de estos, que correspondió al reactivo Donación de sangre, una de las aportaciones de un alumno para explicar su manera de solucionar el problema fue: "Teníamos que ver el texto y luego ver la pregunta que te decía que a qué se refería cuando te decía que necesitaban poner que las jeringas eran de un solo uso y que eran estériles. Entonces tenías que reflexionar, yo sí reflexioné que mucha gente tal vez no dona porque le da miedo contagiarse o algo así, entonces como que hacen hincapié de eso porque abajo vuelven a poner que es seguro. Entonces es para certificar que la donación de sangre era segura".

Durante los citados foros también observamos un gran avance en las aportaciones de los alumnos que denotaban una transferencia de conocimientos. En el del primer reactivo de Matemáticas, Manzanas, cuando la docente les preguntaba la relación que encontraban entre el reactivo y algún tema visto previamente, algunos alumnos respondieron brevemente "El tema de la sucesión". Sin embargo, en uno de los últimos foros de discusión sobre el reactivo La seguridad de los teléfonos móviles, que presenta un artículo sobre este tema, escuchamos aportaciones como: "con Física porque hablaba sobre las ondas que produce el celular" o "con Ciencias porque habla de la salud del que usa el teléfono".

Una de las respuestas que también mostraba una transferencia fue en el reactivo Donación de sangre: "Primero recordé la frase la cual vi en mi clase de Biología y traté 
de relacionarla con mis conocimientos previos del tema. Después recordé los diversos casos sobre la infección del SIDA para elegir que la respuesta correcta es la que establece y que es segura para no contraer ninguna ETS", en la que el alumno habla de haber logrado transferir sus conocimientos adquiridos durante la clase de Biología, específicamente sobre ETS (Enfermedades de Transmisión Sexual), lo que lo llevó a pensar en la necesidad de que los posibles donadores de sangre sepan que no existe ningún riesgo en el procedimiento.

En relación a la concientización realizada por los niños que se equivocaron en alguna respuesta, aunque al inicio de las sesiones ya comunicaban su error, no reflexionaban acerca del motivo del mismo. Sin embargo, en sesiones posteriores, los alumnos realmente reflexionaban sobre aquello que los llevó a una respuesta equivocada, como en el caso del reactivo Manzanas, ya mencionado: "Yo pensé que como las coníferas rodeaban a las manzanas estas siempre serían mayores", respuesta en la que el alumno comunica breve pero asertivamente que imaginó a las coníferas rodeando los manzanos lo cual lo llevó a decidir que las coníferas serían siempre más por estar alrededor de los manzanos, con lo cual dejó claro cuál que fue el origen de su equivocación. Para el mismo reactivo también observamos importantes ejercicios metacognitivos como el siguiente: "Yo en lugar de utilizar las fórmulas empecé a contar lo que había en cada cuadrado, cuántos manzanos y cuántas coníferas y lo saqué mal", donde el alumno reconoce que decidió que sería mejor contar el número de manzanos y de coníferas en lugar de utilizar las fórmulas, por lo cual obtuvo una respuesta incorrecta. Cuando un alumno reflexiona acerca de sus equivocaciones, esto lo lleva a identificar sus áreas de oportunidad, y de esta manera, se esforzará por no volver a cometer los mismos errores (Quinquer, 2008).

Continuando con la concientización de los errores, destacamos las siguientes reflexiones durante el reactivo El gran cañón, que habla de las variaciones de temperatura que existen en el mismo y sus consecuencias, en el que encontramos respuestas como: "Yo no sabía la palabra desmenuzar y en el texto no encontré la respuesta, pero me fui por la primera de las opciones porque pensaba que lo congelado del agua iba a hacer que las rocas se disolvieran". En esta aportación el alumno reconoce que el desconocimiento de cierta terminología provocó su equivocación. También observamos un gran ejercicio metacognitivo en la respuesta: "La saqué equivocada, pero al final no entendía por qué era esa respuesta. Leí la explicación y entendí más o menos lo que era dilatación porque conoces a veces el significado de las palabras pero puedes pensar que hay otro de acuerdo al contexto. Entonces yo pensé que era la de que el hielo pule las rocas porque pulir es como desgastar y yo pensé en la erosión. Asocié pulir, desgaste y erosión. Entonces descarté la de cementar porque dije no se pueden hacer más duras. Luego tenía dudas con las tres respuestas que quedaban. Como la de dilatación no la entendía muy bien la descarté y dejé la del hielo".

Como producto de sus reflexiones, los alumnos también lograron la construcción o el reforzamiento de aprendizajes, que en ocasiones era acerca de habilidades de pensamiento como el refuerzo de su comprensión lectora, o la identificación de palabras clave. En otras respuestas, como: "Aprendí un poco más acerca de la donación de sangre, por ejemplo que los hombres pueden donar hasta cinco veces al año y las mujeres sólo tres y que entre cada donación deben de pasar ocho semanas", el alumno menciona información específica que aprendió sobre el tema. 
La siguiente respuesta contiene tanto elementos metacognitivos como reflexivos que llevaron al alumno a la construcción de conocimientos, durante el reactivo Carpintero, en donde se pedía a los alumnos identificar el diseño del borde de un jardín que podría construirse con determinados metros de madera: "Al principio me parecía totalmente ilógico que una figura en escalera midiera igual o menor a una línea simple de un ángulo recto. Ahorita que me dicen, yo lo había hecho durante mi comprobación y no me di cuenta hasta ahorita. Hice un ángulo recto y luego hice una línea de escalera y tomé las medidas y me dio lo mismo pero en ese momento no me di cuenta que me dio lo mismo. Ahora estoy de acuerdo con las respuestas porque las explicaciones que dieron mis compañeros realmente estuvieron muy bien".

\section{Segunda categoría: Mediación docente}

La mediación docente para el desarrollo de las habilidades de pensamiento y para el logro de aprendizajes pudo ser observada durante los foros de discusión que las maestras dirigieron después de cada uno de los ejercicios reflexivos escritos realizados por los alumnos.

Pudimos constatar la manera en la que ellas fueron incrementando la calidad de sus preguntas y comentarios en los foros con el objetivo de lograr pensamientos más elevados en sus alumnos. Siguiendo a Vigotsky, el maestro es el responsable de promover un ambiente en el aula para que los alumnos puedan nutrirse unos de otros, enfatizando de esta manera el aspecto social del aprendizaje (Presseisen, 2001).

Dewey (2004) ha sido muy claro al enfatizar que es responsabilidad de los docentes esforzarse cada día por crear las condiciones en el aula que impulsen a sus alumnos hacia su aprendizaje y el crecimiento de sus capacidades. Por consiguiente, cuando las sesiones en el aula se convierten en estímulos para el aprendizaje, esto se vuelve un hábito tanto para el docente como para los alumnos y juntos logran su perfeccionamiento.

Las preguntas de las docentes provocaban grandes respuestas en los alumnos ya que lograban que estos reflexionaran en diferentes sentidos. Por ejemplo, el siguiente cuestionamiento metacognitivo de la docente de Ciencias a un alumno: “Cómo lo dedujiste"? "¿Qué te llevó a esa respuesta?" hizo que el alumno pensara metacognitivamente. Como respuesta a esta pregunta el alumno respondió: "Primero yo leí la pregunta y las respuestas. Al principio me pareció que la respuesta correcta era la A porque en la pregunta te decían que cuál era la idea del científico más no la de los alumnos, entonces después leí la información y sí estaba bien. Descarté las demás y subrayé esa. Era la correcta". Los docentes deben ayudar a sus alumnos a desarrollar la metacognición (Ormrod, 2011).

Una pregunta docente que provocó una transferencia de conocimientos fue: “¿Ese tipo de problemas los has usado en la vida cotidiana? ¿Tú lo has hecho? ¿Cuándo?”, que llevó a los alumnos a transferir algún conocimiento que ellos habían adquirido en otro momento, como cambiar dinero de una moneda a otra durante algún viaje, y aplicarlo en un problema. La respuesta del alumno a esta pregunta fue: "Sí, cuando quieres cambiar dinero de una moneda a otra". Siguiendo a Anderson y Krathwohl (2001, citados por Brookhart, 2010), los docentes deben enseñar para la transferencia para lograr que sus alumnos logren una construcción de significados.

Por otro lado, las preguntas docentes también llevaban a que los alumnos tomaran conciencia de algún error cometido y principalmente que aprendieran de este. Una pregunta de la docente de Matemáticas en este sentido fue: "Las personas que no 
respondieron el manzano, ¿en dónde estuvo su error?”, logrando que aquellos alumnos que no habían respondido que los manzanos crecerían más rápidamente, se dieran cuenta del motivo de su equivocación. En este caso un alumno respondió: "Yo solo utilicé la segunda fórmula, la de 8n, no vi la otra y por eso me salió que eran más coníferas que manzanos". Es muy importante para el alumno tomar conciencia sobre las dificultades que tiene para comprender determinado tema o sobre aquellos conocimientos que necesita esforzarse para aprender (Quinquer, 2008). Aquellos alumnos que mencionaron haber respondido correctamente, también se beneficiaron: Quinquer (2008) afirma que cuando un alumno se concientiza sobre aspectos en los que sí se logró un aprendizaje significativo, este aprendizaje será doblemente reforzado.

Las preguntas de las docentes también reforzaban los aprendizajes alcanzados por los alumnos. Un ejemplo de este tipo de cuestionamientos fue el de la docente de Ciencias: “'Alguien sabe qué es la fiebre puerperal?”, obteniendo respuestas como: "Cuando una mujer muere al dar a luz", y "No nos daban una idea exacta de lo que era la fiebre puerperal, solo nos daban información de que las mujeres al embarazarse y dar a luz, morían", provocando que todos los alumnos aprendieran o reforzaran dicho conocimiento.

\section{Tercera categoría: Nivel de desempeño final de los niños}

Dentro de los hallazgos encontramos los siguientes porcentajes de niños que incrementaron los niveles obtenidos entre las pruebas inicial y final de reactivos PISA (Tabla 1).

Tabla 1

Modificación de niveles entre las Pruebas inicial y final para las Competencias científica, matemática y de Comprensión lectora

\begin{tabular}{lllll}
\hline & Científica & Matemática & $\begin{array}{l}\text { Comprensión } \\
\text { lectora }\end{array}$ & General \\
\hline $\begin{array}{l}\text { Alumnos que } \\
\text { incrementaron su } \\
\text { nivel de } \\
\text { desempeño }\end{array}$ & $43 \%$ & $45 \%$ & $45 \%$ & $44 \%$ \\
\hline
\end{tabular}

Nota: Elaboración propia

Otro hallazgo relevante fue que la Institución pasó de un nivel 2.81 a un 3.35 (los resultados contienen decimales por los cálculos realizados para obtener los promedios). Haber alcanzado y superado el nivel 3 en solo veintiuna sesiones es muy gratificante, tanto que de acuerdo a la SEP, su visión para el 2021 debe establecer lo siguiente: "generalizar, como promedio en la sociedad mexicana, las competencias que en la actualidad muestra el nivel 3 de PISA; eliminar la brecha de los niños mexicanos ubicados hoy debajo del nivel 2, y apoyar de manera decidida a quienes están en el nivel 2 y por arriba de este (SEP, 2011).

Para el análisis de las evaluaciones escolares tomamos en cuenta tres momentos: el primero fue mediante los exámenes que habían sido aplicados a los niños pocas semanas antes del iniciar la investigación. El segundo momento correspondió a los exámenes escolares que fueron aplicados cuando los alumnos llevaban algunas semanas de trabajo reflexivo. El tercer momento fue en los exámenes aplicados a los niños dos 
semanas después de concluido el trabajo con los niños. En la Tabla 2 se encuentran los alumnos que mejoraron sus promedios entre los momentos mencionados:

Tabla 2

Alumnos que incrementaron su promedio entre las evaluaciones ocurridas en los tres momentos

\begin{tabular}{lll}
\hline $\begin{array}{l}\text { Alumnos que incrementaron su } \\
\text { promedio entre el primero y el } \\
\text { segundo momento }\end{array}$ & $\begin{array}{l}\text { Alumnos que incrementaron su } \\
\text { promedio entre el segundo y el } \\
\text { tercer momento }\end{array}$ & $\begin{array}{l}\text { Alumnos que incrementaron } \\
\text { su promedio en las dos } \\
\text { mediciones }\end{array}$ \\
\hline $59 \%$ & $32 \%$ & $32 \%$ \\
\hline Nota: elaboración propia & & \\
\hline
\end{tabular}

\section{Discusión y conclusiones}

Una de las conclusiones a la que llegamos fue que los alumnos mostraron una mejoría progresiva en su nivel de reflexión y de argumentación como en el desarrollo de sus capacidades superiores de pensamiento. De acuerdo a Richart y Perkins (2008), para poder desarrollar el pensamiento, este debe volverse visible. Los pensadores efectivos logran externalizar sus pensamientos a través del habla, la escritura o el dibujo.

También concluimos que los alumnos mejoraron tanto en el nivel de desempeño de acuerdo a PISA, así como en sus promedios escolares. En las entrevistas realizadas a las docentes, ellas coincidieron en que los alumnos mejoraron notablemente. Una de las maestras mencionó en su entrevista: "En el último examen parcial ellos mejoraron mucho, de hecho, varios alumnos que siempre reprobaban me sorprendieron por sus buenas calificaciones. Tuvieron mucho que ver los procesos que realizaron en los días que estuvimos trabajando, por ejemplo, cómo analizar las preguntas para poderlas contestar bien".

Pudimos identificar aquellas mediaciones docentes que ayudaron a los alumnos a desarrollar y perfeccionar sus niveles de reflexión y de pensamiento. Vimos los esfuerzos que realizaban para que sus alumnos pudieran llevar sus niveles de razonamiento a otro nivel, para que identificaran sus procesos de pensamiento que realizaron para responder los reactivos, para que identificaran sus conocimientos previos, para que fueran capaces de expresar sus equivocaciones y para que finalmente lograran un aprendizaje significativo.

Logramos también identificar algunos elementos que mostraron su relevancia para que los niños lograran desarrollar y perfeccionar sus capacidades de pensamiento, como la importancia de comprender que el pensamiento conduce al aprendizaje, que el factor rutina es importante para la práctica del pensamiento, que los alumnos deben aprender a hacer visibles sus pensamientos y las conexiones que ocurren en su mente, que los maestros deben realizar preguntas que provoquen pensamientos elevados, que es muy importante que exista una secuencia en el pensamiento, que los alumnos deben poder transferir sus conocimientos, que las interacciones entre los alumnos los conducen hacia el aprendizaje y que estos pueden aprender por medio de la concientización de sus errores.

Pensamos que el desarrollo del pensamiento reflexivo debe apoyarse desde el nivel Preescolar por las siguientes razones: el pensamiento reflexivo es "una neoformación en la edad preescolar que facilita la preparación para la escuela" 
(González-Moreno, Solovieva, y Quintanar, 2009, p. 175), y es importante fomentarlo desde esta edad porque es cuando se "sientan las bases de las capacidades esenciales de aprendizaje", por lo cual el desarrollo de este tipo de pensamiento debiera ser una meta de las escuelas y (Mejía, 2006, citado por González-Moreno et al., 2009, p. 175).

Recomendamos la realización de un estudio similar al realizado con los niños adolescentes, pero con alumnos de Preescolar, para así obtener información acerca de cuáles serían las manifestaciones de un pensamiento reflexivo en los menores y cómo podría saberse que se está en el camino indicado para su desarrollo. Las actividades a realizar con los niños debieran elegirse de acuerdo al desarrollo cognitivo de los preescolares. Proponemos que entonces que se utilice el juego temático de roles, ya que este ha probado ser fundamental para el desarrollo del pensamiento reflexivo de los menores (González-Moreno et al., 2009).

Finalmente, la línea de acción que proponemos a raíz de los hallazgos encontrados será la propuesta de una Herramienta didáctica para el desarrollo y perfeccionamiento de las habilidades superiores de pensamiento que fuimos conformando con los elementos encontrados. El diseño de esta herramienta está pensado para alumnos de los tres niveles de Secundaria, ya que todos se encuentran en la adolescencia, etapa en la que de acuerdo a Henao y Sórzano (2012), ocurren cambios muy importantes en el desarrollo cognitivo de las personas.

\section{Referencias}

Argüelles, D. C., y Nagles, N. (2000). Estrategias para promover procesos de aprendizaje autónomo (Segunda edición). Colombia: Alfaomega colombiana.

Beyer, B. K. (2001). What research says about teaching thinking skills. En A. Costa (Ed.), Developing minds: A resource book for teaching thinking (275-282). Alejandría, VA.: Association for Supervision and Curriculum Development.

Brookhart, S. M. (2010). How to assess higher-order thinking skills in your classroom. Alejandría, VA.: Association for Supervision and Curriculum Development

Brooks, J. G., \& Brooks, M. G. (2001). Becoming a constructivist teacher. In A. Costa (Ed.), Developing minds: A resource book for teaching thinking (101-118). Alejandría, VA.: Association for Supervision and Curriculum Development.

Budsankom, P., Sawangboon, T., Damrongpanit, S., \& Chuensirimongkol, J. (2015). Factors affecting higher order thinking skills of students: A meta-analytic structural equation modeling study. Academic Jorunals Educational Research and Reviews, 10(19), 2640-2652. https://doi.org/10.5897/ERR2015. 2371

Correa, M. E., Castro, F. C., y Lira, H. (2002). Hacia una conceptualización de la metacognición y sus ámbitos de desarrollo. Horizontes Educacionales, 7(1), 5863.

Costa, A. L. (2001a). Developing minds: A resource book for teaching thinking. Alejandría, VA.: Association for Supervision and Curriculum Development.

Costa, A. L. (2001b). Mediating the Metacognitive. En A. Costa (Ed.), Developing minds: A resource book for teaching thinking (57-62). Virginia, E.U.A.: Association for 
Dewey, J. (2004). Experiencia y Educación. Madrid: Biblioteca nueva.

Dewey, J. (2007). Cómo pensamos. Barcelona: Paidós.

González-Moreno, C. X. (2012). Formación del pensamiento reflexivo en estudiantes universitarios. Magis: Revista Internacional de Investigación En Educación, 4(9), 595-617.

González-Moreno, C. X., Solovieva, Y., y Quintanar, L. (2009). La actividad de juego temático de roles en la formación del pensamiento reflexivo en preescolares. Magis. Revista Internacional de Investigación En Educación, 2(3), 173-189.

Henao, M., y Solórzano, B. A. (2012). Una aproximación al desarrollo del pensamiento en el adolescente. Revista Universidad EAFIT, 31(100), 53-60.

Hernández, R., Fernández-Collado, C., y Baptista, P. (2008). Metodología de la Investigación (Cuarta edición). México: McGraw-Hill.

Hmelo, D., \& Ferrari, M. (1997). The problem-based learning tutorial: Cultivating higher order thinking skills. Journal for the Education of the Gifted, 20(4), 401422.

Larrazolo, N., Backhoff, E., y Tirado, F. (2013). Habilidades de razonamiento matemático de estudiantes de educación media superior en México. Revista Mexicana de Investigación Educativa, 18(59), 1137-1163.

Lipman, M. (1998). Pensamiento complejo y educación (Segunda edición). Madrid: Ediciones de la Torre.

Marzano, R. J. (2001). A New Taxonomy of Educational Objectives. En A. Costa (Ed.), Developing minds: A resource book for teaching thinking (181-188). Alejandría, VA.: Association for Supervision and Curriculum Development.

Moore, T. W. (2006). Filosofía de la Educación. México: Trillas.

Muria, I. D., y Damián, M. (2008). Desarrollo de las habilidades del pensamiento en los diferentes niveles educativos. Revista Electrónica de Psicología Iztacala, 11(1), $141-151$.

Nickerson, R., Perkins, D., \& Smith, E. (1985). The teaching of thinking. Hillsdale, N.J.; Lawrence Erlbaum Associates.

Ormrod, J. E. (2011). Aprendizaje humano (Cuarta edición). España: Pearson Prentice Hall.

Perkins, D. N., \& Salomon, G. (2001). Teaching for transfer. En A. Costa (Ed). Developing minds: A resource book for teaching thinking (370-378). Alejandría, VA.: Association for Supervision and Curriculum Development.

Presseisen, B. Z. (2001). Thinking skills: meanings and models revisited. En A. Costa (Ed.), Developing minds: A resource book for teaching thinking (47-53). Alejandría, VA.: Association for Supervision and Curriculum Development.

Quinn, M. (1990). Qualitative evaluation and Research methods (Second edition). Newbury Park: Sage Publications.

Quinquer, D. (2008). Modelos y enfoques sobre la evaluación el modelo comunicativo. En Evaluación como ayuda al aprendizaje (13-19). Barcelona: Graó. 
Resnick, L. B. (2001). Making America smarter: The real goal of school reform. En A. Costa (Ed.), Developing minds: A resource book for teaching thinking (3-6). Alejandría, VA: Association for Supervision and Curriculum Development.

Richart, R., \& Perkins, D. (2008). Making thinking visible. Educational leadership, 65(5), 57-61.

Rohana. (2015). The Enhancement of Student's Teacher Mathematical Reasoning Ability through Reflective Learning. Journal of Education and Practice, 6(20), 108-115.

Saiz, C. (2002). Enseñar o aprender a pensar. Escritos de Psicología, (6), 53-71.

Sánchez, M. A. (2002). La investigación sobre el desarrollo y la enseñanza de las habilidades de pensamiento. Revista Electrónica de Investigación Educativa, $4(1), 1-32$.

SEP, Secretaría de Educación Pública. (2011). Plan de estudios 2011. Educación básica. México: SEP, Secretaría de Educación Pública.

Song, H. D., Koszalka, T., \& Grabowski, B. (2005). Exploring Instructional Design Factors Prompting Reflective Thinking in Young Adolescents. Canadian Journal of Learning and Technology, 31(2), 1-17.

Fecha de recepción: 20/04/2017

Fecha de revisión: 12/10/2017

Fecha de aceptación: 21/10/2017 